\title{
BMJ Carbidopa/levodopa dose elevation and open safety concerns in Parkinson's patients: a cross-sectional and cohort design
}

\author{
David W Brodell, ${ }^{1}$ Nicole T Stanford, ${ }^{1}$ Charles E Jacobson, ${ }^{1}$ Peter Schmidt, ${ }^{2}$ \\ Michael S Okun ${ }^{1}$
}

To cite: Brodell DW, Stanford NT, Jacobson CE et al. Carbidopa/levodopa dose elevation and safety concerns in Parkinson's patients: a cross-sectional and cohort design. BMJ Open 2012;2:e001971. doi:10.1136/bmjopen-2012001971

- Prepublication history for this paper are available online. To view these files please visit the journal online (http://dx.doi.org/10.1136/ bmjopen-2012-001971).

Received 16 August 2012 Revised 26 October 2012 Accepted 29 October 2012

This final article is available for use under the terms of the Creative Commons Attribution Non-Commercial 2.0 Licence; see

http://bmjopen.bmj.com

\footnotetext{
${ }^{1}$ Department of Neurology, University of Florida Center for Movement Disorders and Neurorestoration, Gainesville, Florida, USA

${ }^{2}$ National Parkinson

Foundation, Miami, Florida, USA
}

\section{Correspondence to} Dr Michael S Okun; okun@neurology.ufl.edu

\begin{abstract}
Objective: Sinemet, a combination drug containing carbidopa and levodopa is considered the gold standard therapy for the treatment of Parkinson's disease (PD). When approved by the Food and Drug Administration (FDA) in 1988, a maximum daily dosage limit of $800 \mathrm{mg}$ (eight tablets) of the 25/100 carbidopa/levodopa formulation was introduced. Overall, the FDA approval was a historic success; however, the pill limit has been hardcoded into many online medical record systems. This study investigates the $800 \mathrm{mg}$ threshold by using a prospectively collected database of patient information.
\end{abstract}

Design: A retrospective cohort study: (Part I) crosssectional, (Part II) longitudinal.

Setting and participants: PD patients at a Movement Disorders Center in a large academic, tertiary medical setting.

Outcome measures: An analysis was performed using carbidopa/levodopa at dosages below and above the $800 \mathrm{mg}$ threshold. A secondary analysis was then performed using two consecutive clinic visits to determine the effects of crossing the $800 \mathrm{mg}$ threshold. Comparisons were made on standardised scales.

Results: There was no significant difference in motor, mood and quality-of-life scores in patients consuming below and above the $800 \mathrm{mg}$ carbidopa/levodopa threshold, though a mild worsening in dyskinesia duration was noted without worsening in dyskinesia pain and disability. In PD patients who crossed the $800 \mathrm{mg}$ threshold between two consecutive clinic visits, a significant improvement in depressive symptoms and quality-of-life measures was demonstrated, and in these patients there was no worsening of motor fluctuations or dyskinesia.

Conclusions: The data suggest that PD patients have the potential for enhanced clinical benefits when eclipsing the $800 \mathrm{mg}$ carbidopa/levodopa threshold. Many patients will likely need to eclipse the $800 \mathrm{mg}$ threshold and pharmacies and insurance companies should be aware of the requirements that may extend beyond approval limits.

\section{INTRODUCTION}

Carbidopa/levodopa has been the most effective first-line pharmacological therapy in

\section{ARTICLE SUMMARY}

Article focus

- To investigate the effects of crossing the Food and Drug Administration's Sinemet dose limit in a cohort of patients with Parkinson's disease.

Key messages

- Simple comparisons provide evidence that it is safe, and additionally there are important potential benefits to crossing the threshold.

- A cross-sectional investigation did not show significant differences in motor, mood and quality-of-life scores in patients consuming below and above the $800 \mathrm{mg}$ carbidopa/levodopa threshold.

- A longitudinal analysis showed significant improvement in depressive symptoms and quality-of-life measures with no worsening of motor fluctuations or dyskinesia when crossing the threshold.

Strengths and limitations of this study

- A key strength of this study is its use of statistical comparisons to investigate an important care-related issue. The results of this study will provide evidence for patients who may be denied medications by pharmacies or insurance providers.

- Other safety measures will need to be documented in future studies including hallucinations, nausea, daytime sleepiness and orthostatic hypotension to confirm a favourable safety record when eclipsing the $800 \mathrm{mg}$ threshold. Also, the single-centre setting and retrospective design limit the generalisability of these results.

the treatment of Parkinson's disease (PD). ${ }^{1} 2$ When approved by the Food and Drug Administration (FDA) in 1988, a maximum dosage threshold of eight $100 \mathrm{mg}$ pills/day was written in as the limit. This limit was based on animal studies which revealed, 'a significant proportion of infant rats of both sexes are expected to die at a dose of $800 \mathrm{mg} / \mathrm{kg}$. ${ }^{3}$ FDA authorisation was a historic success; however, the carbidopa/levodopa limit has resulted in important issues for patients and for clinicians. The limit 
commonly shows up in many online medical record systems, pharmacies and websites. The limit has also been widely cited in coverage decisions by insurance providers seeking to limit the number of tablets dispensed to a single PD sufferer.

It is well known and appreciated that many patients, especially those with advanced $\mathrm{PD}$, will require frequent doses in excess of $800 \mathrm{mg}$ a day. The current limit sets up a potentially worrisome scenario for patients requiring more than $800 \mathrm{mg}$ a day of dopamine replacement. Carbidopa/levodopa plays an important role in the management of PD by symptomatically addressing many of its disabling disease manifestations including tremor, rigidity, bradykinesia, postural instability, gait issues and also some non-motor features. ${ }^{2}$ Under-medication can contribute to destabilising the clinical features of the disease and could lead to an increased risk of falls and aspiration pneumonia. ${ }^{4}$ As PD progresses, many patients eclipse eight $100 \mathrm{mg}$ pills a day, and will ultimately require greater amounts of levodopa to be taken at more frequent medication intervals (eg, every 2 hours).

There have been few studies that have specifically investigated the carbidopa/levodopa dose-response. ${ }^{5-7}$ No study has specifically addressed the $800 \mathrm{mg}$ threshold question. In this investigation, we examined the clinical consequences of crossing the $800 \mathrm{mg}$ threshold. We hypothesised that crossing the $800 \mathrm{mg}$ threshold would lead to general improvement in clinical symptoms without adding significant safety issues.

\section{METHODS}

Data were obtained following informed consent and through the use of the Institutional Review Board-approved protocol for the University of Florida-INFORM (Interdisciplinary Florida Registry and Movement Disorders) database. All patients treated with carbidopa/ levodopa (Sinemet, Stalevo, Parcopa) from July 2002 to December 2009 were included. Inclusion criteria required a diagnosis of idiopathic PD using UK Brain Bank criteria ${ }^{8}$ according to a movement disorders trained specialist. Patients with deep brain stimulation devices were excluded. It was required that the Unified Parkinson's Disease Rating Scale (UPDRS), the Parkinson's Disease Quality of Life Questionnaire (PDQ-39) and the Beck Depression Inventory (BDI) were administered to all patients at each clinical visit. The strict criteria were employed to limit variability in the comparisons.

The first analysis was between-group, examining those patients above and those patients below a daily levodopa dosage threshold of $800 \mathrm{mg}$. Each group was limited to Hoehn-Yahr stages 2, 2.5 and 3 and to a disease duration of 5-10 years. Figure 1 summarises the data used in this analysis, and the reasons for exclusion. The threshold was determined at each subject's first visit to the University of Florida Center for Movement Disorders and Neurorestoration. The mean levodopa dosage, Hoehn-Yahr Parkinson stage and disease duration for

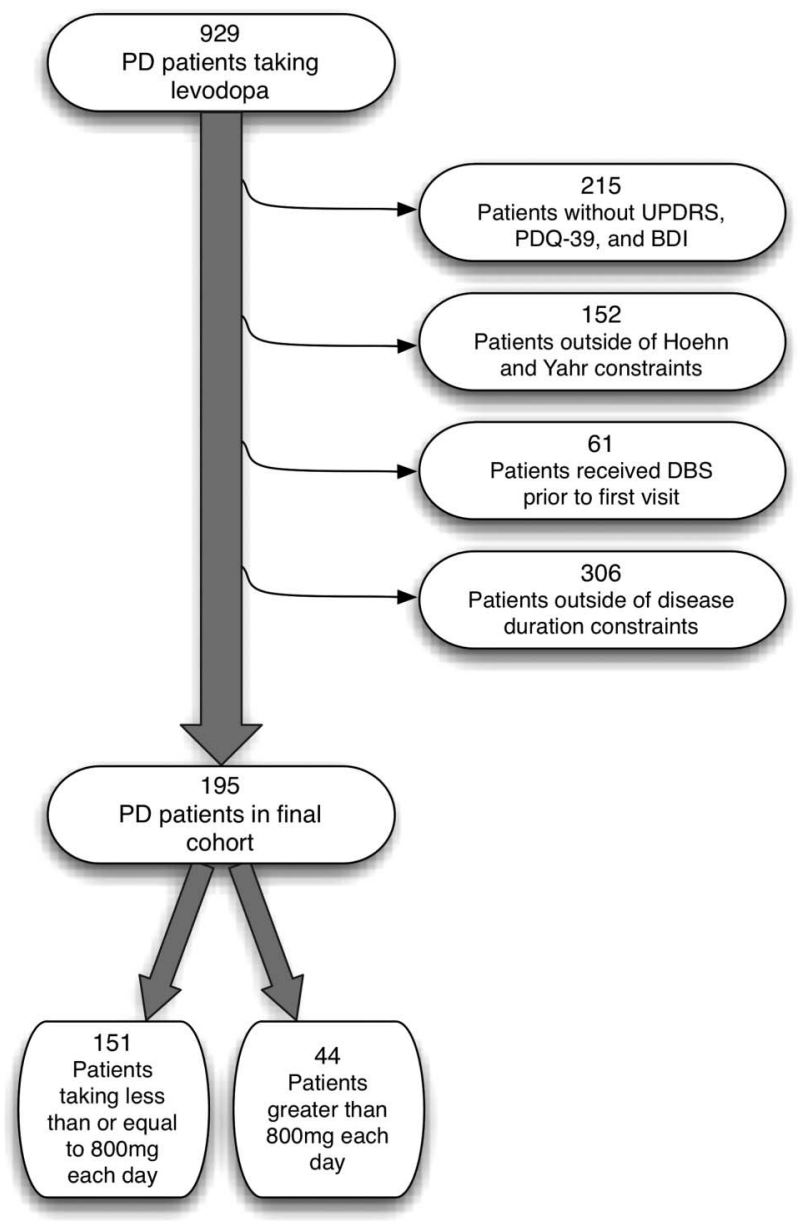

Figure 1 Selection of patients from the database using strict inclusion/exclusion criteria.

the two groups (above and below $800 \mathrm{mg}$ ) were documented. In addition, the UPDRS Part III motor score in the 'on' dopaminergic medication state, the UPDRS Part IV motor fluctuations and dyskinesia subscores, the PDQ-39 quality-of-life score, and the BDI depression score were used in the analysis, and in the between-groups comparison. The Wilcoxon rank-sum test was used to detect significant differences between groups for the BDI, PDQ-39 and UPDRS motor scales. The $\chi^{2}$ test was used to identify differences in agonist use, and Fisher's exact test was utilised for the categorical dyskinesia questions. In each dyskinesia question, categories 2-4 were combined. In addition, the levodopa dosage, the levodopa equivalent $\operatorname{dose}^{9}$ and the use of dopamine agonists were documented. The disease characteristics for each group have been summarised in table 1 .

A second analysis was performed using patients who crossed the threshold (moving above $800 \mathrm{mg}$ ) when tracked for two consecutive clinic visits. For this analysis, the following criteria were employed: (1) at the first clinic visit the dosage had to be below or equal to $800 \mathrm{mg}$ and (2) at the second clinic visit the dosage had to be above $800 \mathrm{mg}$. The second visit was required to be within 1 year of the first visit in order to minimise the 
Table 1 Disease characteristics above and below levodopa threshold in a between-group analysis

\begin{tabular}{|c|c|c|c|}
\hline & Less than or equal to $800 \mathrm{mg}$ & Greater than $800 \mathrm{mg}$ & p Value \\
\hline Patient count & 151 & 44 & NA \\
\hline Age (years) & $72.83(10.25)$ & $72.39(10.54)$ & NS \\
\hline Disease duration (years) & $7.11(1.65)$ & $7.64(1.66)$ & NS \\
\hline Agonist present, count ${ }^{\star}$ & $70,46.4 \%$ & $22,50.0 \%$ & NS \\
\hline Dose (mg) & 470.53 (166.96) & $1212.77(352.80)$ & $<0.0001$ \\
\hline LED (mg) & 532.49 (204.29) & 1267.28 (361.95) & $<0.0001$ \\
\hline UPDRS Part III & $29.40(9.87)$ & $28.95(7.50)$ & NS \\
\hline PDQ-39 QOL & $27.43(16.55)$ & $30.06(13.34)$ & NS \\
\hline BDI & $9.79(7.37)$ & $9.95(5.57)$ & NS \\
\hline Dyskinesia duration, Q32 & & & 0.0003 \\
\hline $0 \%$ & $99(69.2 \%)$ & $17(39.5 \%)$ & \\
\hline $1-25 \%$ & 27 (18.9\%) & $21(48.8 \%)$ & \\
\hline $26-100 \%$ & $17(11.9 \%)$ & $5(11.6 \%)$ & \\
\hline Dyskinesia disability, Q33 & & & 0.724 \\
\hline None & $120(83.3 \%)$ & $38(88.4 \%)$ & \\
\hline Mild & $13(9.0 \%)$ & $2(4.7 \%)$ & \\
\hline Moderate, severe & $11(7.6 \%)$ & $3(7.0 \%)$ & \\
\hline Dyskinesia pain, Q34 & & & 0.787 \\
\hline None & $131(91.6 \%)$ & $38(88.4 \%)$ & \\
\hline Slight & $4(2.8 \%)$ & $1(2.3 \%)$ & \\
\hline Moderate, severe & $8(5.6 \%)$ & $4(9.3 \%)$ & \\
\hline Percent of day off medication, Q39 & & & 0.081 \\
\hline $0 \%$ & $61(43.0 \%)$ & $11(25.6 \%)$ & \\
\hline $1-25 \%$ & $55(38.7 \%)$ & $19(44.2 \%)$ & \\
\hline $26-100 \%$ & $26(18.3 \%)$ & $13(30.2 \%)$ & \\
\hline
\end{tabular}

Standard deviation or percent composition in parenthesis.

NS, not statistically significant; NA, not applicable.

*Number of records where agonist is present.

effects of disease progression. The UPDRS Part III, UPDRS Part IV, PDQ-39, BDI and dyskinesia subscores from each clinic visit were compared using the paired Wilcoxon signed-rank test. The Cochran $Q$ test was used to identify differences in the dyskinesia questions, and the McNemar test was used for agonist analysis. Table 2 summarises the findings.

The $\alpha$ was set at $\mathrm{p}<0.01$ because multiple comparisons were performed. IBM SPSS Statistics V.20 was used for all analysis.

\section{RESULTS}

The between-group analysis revealed that 195 PD patients met inclusion criteria (151 were below the $800 \mathrm{mg}$ threshold and 44 above). The disease characteristics have been summarised in table 1 . The mean levodopa doses were $470.5 \mathrm{mg}$ for the below-threshold group and $1212.8 \mathrm{mg}$ for the above-threshold group. The LED (which takes into account all dopaminergic drugs) was also calculated. ${ }^{9}$

Dyskinesia information was available for 185/195 patients $(94.9 \%)$. The Wilcoxon rank-sum test revealed worsening in dyskinesia duration as measured by question 32 from the UPDRS Part IV scale $(p<0.01)$ for the above $800 \mathrm{mg}$ threshold group. Though dyskinesia duration slightly worsened, dyskinesia pain and dyskinesia disability did not significantly differ between groups. The remaining scales (UPDRS Part III, PDQ-39 QOL, BDI) did not reveal significant between group differences.

A longitudinal analysis between two clinic visits was then performed. A sample of 28 patients drawn from the above group who crossed the $800 \mathrm{mg}$ threshold from visits 1 to 2 was identified. The second appointment was an average of 229 days from the first, with a standard deviation of 95 . The disease characteristics of the longitudinal cohort have been summarised in table 2 . Utilising the Wilcoxon signed rank test, improvements in QOL $(\mathrm{p}<0.01)$ and depressive symptoms according to BDI $(p<0.001)$ were revealed in the group that exceeded $800 \mathrm{mg}$ (figure 2A,B). Changes in motor score and dyskinesia categories were not significantly different between groups.

\section{DISCUSSION}

The data from this study revealed that eclipsing the $800 \mathrm{mg}$ levodopa threshold was associated with mild worsening of dyskinesia symptoms without worsening disability, while maintaining benefit in motor, mood and quality-of-life scales. Further, a longitudinal analysis performed between two consecutive clinic visits revealed that depressive symptoms and quality of life improved 
Table 2 Disease characteristics above and below levodopa threshold in a longitudinal analysis drawn from consecutive patients

\begin{tabular}{|c|c|c|c|}
\hline & Visit 1 & Visit 2 & p Value \\
\hline Count & 28 & - & NA \\
\hline Age (years) & $72.29(10.02)$ & - & NS \\
\hline Disease Duration (years) & $6.93(3.85)$ & - & NS \\
\hline Agonist present & $14,50 \%$ & $12,42.9 \%$ & NS \\
\hline Dose $(\mathrm{mg})$ & $585.29(166.38)$ & $1102.50(237.15)$ & $<0.0001$ \\
\hline LED (mg) & $664.23(239.54)$ & $1162.11(245.11)$ & $<0.0001$ \\
\hline UPDRS Part III & $31.86(10.68)$ & $28.75(8.36)$ & NS \\
\hline PDQ-39 QOL & 32.79 (17.69) & $27.69(21.31)$ & $<0.01$ \\
\hline $\mathrm{BDI}$ & $12.82(9.44)$ & $10.04(8.47)$ & $<0.01$ \\
\hline Dyskinesia duration, Q32 & & & 0.572 \\
\hline $0 \%$ & $19(51.4 \%)$ & $18(48.6 \%)$ & \\
\hline $1-25 \%$ & $3(37.5 \%)$ & $5(62.5 \%)$ & \\
\hline $26-100 \%$ & $4(44.4 \%)$ & $5(55.6 \%)$ & \\
\hline Dyskinesia disability, Q33 & & & 0.572 \\
\hline None & $22(48.9 \%)$ & $23(51.1 \%)$ & \\
\hline Mild & $2(40.0 \%)$ & $3(60.0 \%)$ & \\
\hline Moderate, severe & $3(60.0 \%)$ & $2(40.0 \%)$ & \\
\hline Dyskinesia pain, Q34 & & & NAt \\
\hline None & $26(50.0 \%)$ & $26(50.0 \%)$ & \\
\hline Slight & & $2(100.0 \%)$ & \\
\hline Moderate, severe & & & \\
\hline Percent of day OFF Medication, Q39 & & & 0.521 \\
\hline $0 \%$ & $7(53.8 \%)$ & $6(46.2 \%)$ & \\
\hline $1-25 \%$ & $13(50.0 \%)$ & $13(50.0 \%)$ & \\
\hline $26-100 \%$ & $6(40.0 \%)$ & $9(60.0 \%)$ & \\
\hline
\end{tabular}

Standard deviation in parenthesis

NS, not statistically significant; NA, not applicable

${ }^{*}$ Number of records where agonist is present

†Dyskinesia Q34 has only one group at visit 1-the associated test could not be carried out.

when crossing the threshold. Early in the course of PD treatment, patients typically use two or three $100 \mathrm{mg}$ pills a day to maintain what has been referred to as an 'on' medication state. This has also been referred to as the 'honeymoon period'. Tremors, stiffness, slowness and other symptoms typically improve during the 'on' medication state. However, following 5 years of PD duration, the majority of patients experience a wearingoff effect between their dosages, and also on-off fluctuations. In addition, many patients experience hyperkinetic movements referred to as dyskinesia. ${ }^{9}$ Immediate-release levodopa is associated with a short half-life (approximately $90 \mathrm{~min}$ ), and intraneuronal buffering most likely contributes to near-continuous stimulation between dosages. ${ }^{10}$ As PD progresses, however, this buffering capacity seems to diminish with the loss of nigrostriatal terminals, and plasma levels fluctuate more frequently. ${ }^{10} 9$ As the disease duration increases, levodopa doses typically increase, and must be administered at closer intervals in order to avoid motor fluctuations. Some PD patients will actually require doses to be administered as frequently as every 2 hours all day and possibly during the night. It is also important to note that similar doses of different standard oral carbidopa/ levodopa formulations will likely manifest different clinical and pharmacological effects. Further, continuous intrajejunal infusion of levodopa has been observed to have a different benefit and side effect profile relative to traditional oral levodopa administered at similar doses. In one study, an intrajejunal levodopa infusion of 1996 $\pm 675 \mathrm{mg} /$ day (range, $1100-3204 \mathrm{mg} /$ day) led to significant improvement with few side effects. ${ }^{11}$ This dosage was well above the $800 \mathrm{mg}$ FDA limit, and this provides further evidence that the pill limit should be revisited.

The current study used two methodologies to examine key disease characteristics of patients below and above the $800 \mathrm{mg}$ levodopa threshold set by the FDA in 1988 . PD patients in our study tolerated a levodopa dosage increase to above the $800 \mathrm{mg}$ threshold, though there was an expected modest increase in dyskinesia duration, but not disability with escalation of dosages in the between-group scenario. Interestingly, patients in the longitudinal analysis did not worsen in any of the dyskinesia categories, highlighting the notion that careful medication titration by experienced practitioners can limit adverse side effects of dopaminergic therapy.

No study has followed up on the animal toxicity data that were quoted in the FDA approval letter for carbidopa/levodopa. ${ }^{3}$ There are however, several important published observations on carbidopa/levodopa. An animal study using hemiparkinsonian monkeys observed that optimal antiparkinsonian effects occurred at 

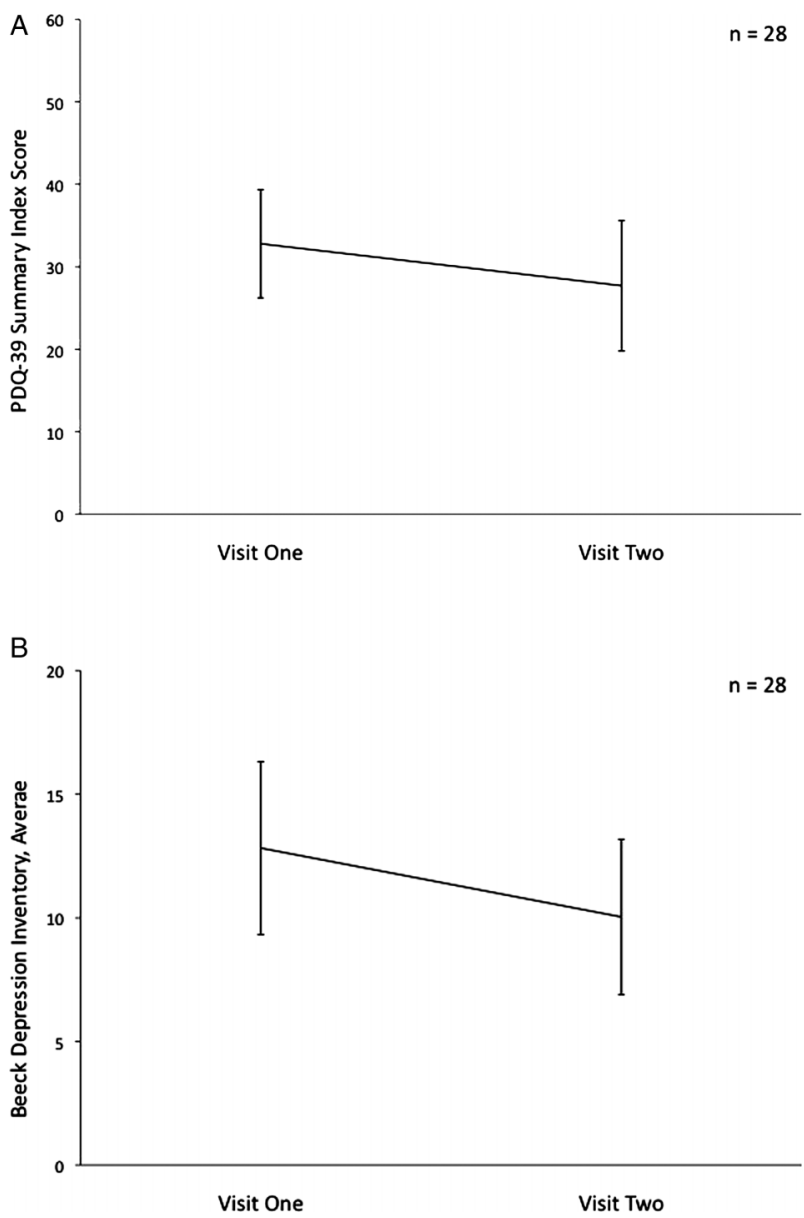

Figure 2 (A) Improvement in quality of life when increasing above the $800 \mathrm{mg}$ levodopa threshold. (B) Improvement in depressive symptoms when increasing above the $800 \mathrm{mg}$ levodopa threshold.

dosages of $1000-2000 \mathrm{mg} /$ day. $^{6}$ Similar to the findings in our study, the recent ELLDOPA trial revealed that escalating doses of carbidopa/levodopa resulted in better symptomatic improvement, but also the potential for increased motor fluctuations. ${ }^{5}$ In another study, a large daily levodopa dose range was observed to be necessary to control symptoms in individual patients. Analysis of a low-dose (median daily dose of $400 \mathrm{mg}$ ) and a high-dose group (median daily dose of $1017 \mathrm{mg}$ ) revealed 'no clear differences between the groups' and no difference in dyskinesia. ${ }^{7}$ No study in the literature has shown a significant toxicity or safety issue when eclipsing the $800 \mathrm{mg}$ threshold, although higher doses have been associated with lethargy, psychosis, othostasis and nausea. ${ }^{2}$ These side effects when encountered, however, can be addressed by dose, interval or addition of other medications.

One important tenet in the treatment of PD is that most patients prefer to be in the 'on' dopaminergic medication state, even if dyskinesia is present. ${ }^{10}$ Some PD patients have been noted to be anosognosic or unaware of their dyskinesia, and only the caregiver may notice the hyperkinetic state. ${ }^{12}$ Many strategies have been employed to limit motor fluctuations, but as disease duration increases, typically the medication interval decreases, and the levodopa dosage will quickly escalate above the $800 \mathrm{mg}$ threshold in the majority of sufferers. ${ }^{13}$

One limitation of our study was the exclusion of a large number of PD patients who were on levodopa replacement therapy. This exclusion was purposeful, as we sought to narrow the groups above and below $800 \mathrm{mg}$ to be comparable on all major disease state measures. A further limitation was that the scales were drawn from a clinical research database, which through a single centre utilised multiple independent fellowship trained movement disorders specialists to perform the ratings. All specialists were specifically trained with the Movement Disorders Society UPDRS training tape; however, this process could have introduced variability. The patients were, however, seen by the same neurologist from visit to visit. In addition, the lack of blinding may have introduced bias. Additionally, the measurement of motor fluctuations and dyskinesias was limited by the use of categorical questions from the UPDRS Part IV, rather than by the gold standard 2-day on-off fluctuation diary. ${ }^{14}$ Also, it should be considered that higher dosages of levodopa are often frequently paired with lower dosages of dopamine agonists and this could have affected the findings. Finally, in future studies, other safety measures should be documented including hallucinations, nausea, daytime sleepiness, orthostatic hypotension and even death as documentation of a favourable safety record would also support eclipsing the $800 \mathrm{mg}$ threshold. The establishment of a multicenter prospective database to comprehensively track PD medications, dosages and side effects could be useful to the field.

In conclusion, the data suggest that PD patients may achieve an enhanced benefit when eclipsing the $800 \mathrm{mg}$ levodopa threshold that has been imposed by the FDA. The treatment of advanced PD will frequently require the clinician to administer more than $800 \mathrm{mg}$ of levodopa per day, and therefore we conclude that it would be reasonable for the FDA to consider revision of its recommendation. This revision would enable online medical record systems, pharmacies and insurance carriers to act on the critical need that many PD patients have for more than eight carbidopa/levodopa tablets each day.

Acknowledgements We would like to acknowledge the support of the National Parkinson Foundation Center of Excellence and the UF INFORM database.

Contributors PS and MO were involved in study conception and design; $\mathrm{CJ}$ and DB were involved in acquisition of data; DB and MO carried out primary analysis, organisation and interpretation; DB and NS were involved in primary writing; $\mathrm{MO}, \mathrm{DB}, \mathrm{CJ}$ and $\mathrm{PS}$ performed critical revisions and MO supervised the study.

Funding This study was supported financially by the grant support provided by the National Parkinson Foundation Center of Excellence located at the University of Florida, Gainesville. MO serves as a consultant for the National Parkinson Foundation, and has received research grants from NIH, NPF, the Michael J. Fox Foundation, the Parkinson Alliance, Smallwood Foundation and the UF Foundation. MO has in the past $>36$ months received no support from industry. MO has received royalties for publications with Demos, Manson and 
Cambridge (movement disorders books). MO has participated in CME activities on movement disorders sponsored by the USF CME office, Prime, Delaware Media, Journal Watch, PeerView and by Vanderbilt University. The institution and not MO receives grants from Medtronic and ANS/St Jude, and the $\mathrm{PI}$ has no financial interest in these grants. MO has participated as a site $\mathrm{PI}$ and/or co-I for several $\mathrm{NIH}$, foundation, and industry sponsored trials over the years, but has not received honoraria. Publication of this article was funded in part by the University of Florida Open-Access Publishing Fund.

Competing interests DB, NS, CJ and PS have nothing to disclose.

Ethics approval The University of Florida Institutional Review Board (IRB-01).

Provenance and peer review Not commissioned; externally peer reviewed.

Data sharing statement Please contact CJ at jacobson@neurology.ufl.edu for access

\section{REFERENCES}

1. Jain L, Benko R, Safranek S. Clinical inquiry. Which drugs work best for early Parkinson's disease? J Fam Pract 2012;61:106-8.

2. Miyasaki JM, Martin W, Suchowersky O, et al. Practice parameter: initiation of treatment for Parkinson's disease: an evidence-based review: report of the Quality Standards Subcommittee of the American Academy of Neurology. J Neurol 2002;58:11-17.

3. Merck \& Co., Inc. United States. Food and Drug Administration. Sinemet 1988.

4. Temlett JA, Thompson PD. Reasons for admission to hospital for Parkinson's disease. J Intern Med 2006;36:524-6.
5. Fahn S. Parkinson disease, the effect of levodopa, and the ELLDOPA Trial. Earlier vs later L-DOPA. Arch Neurol 1999;56:529-35.

6. Kurlan R, Kim MH, Gash DM. Oral levodopa dose-response study in MPTP-induced hemiparkinsonian monkeys: assessment with a new rating scale for monkey parkinsonism. Mov Disord 1991;6:111-18.

7. Nyholm D, Karlsoon E, Lundberg M, et al. Large differences in levodopa dose requirement in Parkinson's disease: men use higher doses than women. Eur J Neurol 2010;17:260-6.

8. Hughes AJ, Daniel SE, Ben-Schlomo Y, et al. The accuracy of diagnosis of parkinsonian syndromes in a specialist movement disorder service. Brain 2002;125:861-70.

9. Hobson DE, Lang AE, Martin WR, et al. Excessive daytime sleepiness and sudden-onset sleep in Parkinson disease: a survey by the Canadian Movement Disorders Group. JAMA 2002;287:455-63.

10. Poewe W, Antonini A, Zijlmans JC, et al. Levodopa in the treatment of Parkinson's disease: an old drug still going strong. Clin Interv Aging 2010;5:229-38.

11. Reddy P, Martinez-Martin P, Rizos A, et al. Intrajejunal levodopa versus conventional therapy in Parkinson disease: motor and nonmotor effects. Clin Neuropharmacol 2012;35:205-7.

12. Lu LH, Barrett AM, Cibula JE, et al. Dissociation of anosognosia and phantom movement during the Wada test. $J$ Neurol Neurosurg Psychiatry 2000;69:820-3.

13. Khlebtovsky A, Rigbi A, Melamed E, et al. Patient and caregiver perceptions of the social impact of advanced Parkinson's disease and dyskinesias. J Neural Transm 2012;119:1367-71.

14. Papapetropoulos SS. Patient diaries as a clinical endpoint in Parkinson's disease clinical trials. CNS Neurosci Ther 2012;18:380-7. 